\title{
Image-based 3D modeling and validation of radiofrequency interstitial tumor ablation using a tissue-mimicking breast phantom
}

\author{
Zhigang Wang - Isshaa Aarya - Mariana Gueorguieva - Dun Liu • \\ Hongyan Luo - Luigi Manfredi - Lijun Wang - Donald McLean • \\ Stuart Coleman · Stuart Brown · Alfred Cuschieri
}

Received: 10 January 2012 / Accepted: 29 May 2012 / Published online: 12 June 2012

(C) CARS 2012

\begin{abstract}
Purpose Minimally invasive treatment of solid cancers, especially in the breast and liver, remains clinically challenging, despite a variety of treatment modalities, including radiofrequency ablation (RFA), microwave ablation or highintensity focused ultrasound. Each treatment modality has advantages and disadvantages, but all are limited by placement of a probe or US beam in the target tissue for tumor ablation and monitoring. The placement is difficult when the tumor is surrounded by large blood vessels or organs. Patientspecific image-based 3D modeling for thermal ablation simulation was developed to optimize treatment protocols that improve treatment efficacy.

Methods A tissue-mimicking breast gel phantom was used to develop an image-based 3D computer-aided design (CAD) model for the evaluation of a planned RF ablation. First, the tissue-mimicking gel was cast in a breast mold to create a 3D breast phantom, which contained a simulated solid tumor. Second, the phantom was imaged in a medical MRI scanner using a standard breast imaging MR sequence. Third, the MR images were converted into a 3D CAD model using commercial software (ScanIP, Simpleware), which was input into another commercial package (COMSOL Multiphysics) for RFA simulation and treatment planning using a finite element method (FEM). For validation of the model, the breast phantom was experimentally ablated using a commercial (RITA) RFA electrode and a bipolar needle with an electrosurgi-
\end{abstract}

Z. Wang $(\bowtie) \cdot$ I. Aarya $\cdot$ M. Gueorguieva $\cdot$ D. Liu ·

H. Luo - L. Manfredi · L. Wang · D. McLean ·

S. Coleman $\cdot$ S. Brown $\cdot$ A. Cuschieri

Institute for Medical Science and Technology, University of Dundee,

Wilson House, 1 Wurzburg Loan, Dundee DD2 1FD, UK

e-mail: z.z.wang@dundee.ac.uk

A. Cuschieri

e-mail: a.cuschieri@dundee.ac.uk cal generator (DRE ASG-300). The RFA results obtained by pre-treatment simulation were compared with actual experimental ablation.

Results A 3D CAD model, created from MR images of the complex breast phantom, was successfully integrated with an RFA electrode to perform FEM ablation simulation. The ablation volumes achieved both in the FEM simulation and the experimental test were equivalent, indicating that patientspecific models can be implemented for pre-treatment planning of solid tumor ablation.

Conclusion A tissue-mimicking breast gel phantom and its MR images were used to perform FEM 3D modeling and validation by experimental thermal ablation of the tumor. Similar patient-specific models can be created from preoperative images and used to perform finite element analysis to plan radiofrequency ablation. Clinically, the method can be implemented for pre-treatment planning to predict the effect of an individual's tissue environment on the ablation process, and this may improve the therapeutic efficacy.

Keywords Radiofrequency ablation - Image-based 3D modeling $\cdot$ FEM simulation $\cdot$ Cancer treatment

\section{Introduction}

Death from invasive cancer remains one of the leading causes of human mortality and underlies the extensive research for the development of more effective therapies to improve survival. The minimally invasive nature of thermal ablation (the heating of tissue to induce death) makes it a popular choice for cancer treatment especially when surgical resection is not possible or contraindicated [1,2]. However, the clinical application of minimally invasive treatment modalities [3] 
such as radiofrequency ablation (RFA), microwave ablation (MWA) or high-intensity focused ultrasound (HIFU) remains challenging as the efficacy depends on accurate placement of the probe or focusing the HIFU at the target tissue to provide the necessary volumetric heating, which in turn is influenced by the surrounding environment, for example, large blood vessels causing a heat-sink effect, or adjacent organs that have to be spared. The problem is further confounded by the difficulty of monitoring the ablation process itself $[4,5]$. Patient-specific image-based 3D modeling could resolve these problems by permitting pre-treatment planning of the simulated thermal ablation based on preoperative images of the lesion [6].

Radiofrequency ablation (RFA) is a proven, effective method for targeted tumor ablation without causing significant damage to surrounding healthy tissues [7]. RFA is based on the principle that radiofrequency currents induce heating in the tissue. If the temperature of the tumor is raised above $45^{\circ} \mathrm{C}$ for $4 \mathrm{~min}$, thermal necrosis of the lesion ensues. Radiofrequency currents are introduced in the tumor tissue either percutaneously or by a laparoscopic approach. More clinical trials are currently being conducted and mathematical computational models developed to further understand the ablation process and the role of the surrounding tissue and its influence on heat distribution [8]. Most of the reported computational models $[9,10]$ are simplified geometric systems that, though good enough to estimate ablation in FEM studies, do not model the effect of the tissue environment surrounding the tumor.

Human anatomical 3D computer-aided design (CAD) models can be used to perform more complex studies on ablation, which incorporate the surrounding healthy tissues in addition to the tumor. These models can be created by employing a patient's magnetic resonance (MR) or computed tomography (CT) image slices [11] to construct representations of the actual cancer and its surrounding environment. Such models should improve understanding of the thermal ablation process and could help the surgeon to plan the ablation procedure based on individual patient requirements, thereby improving patient outcome [12].

As patient data and images were not available for the study, we used a tissue-mimicking gel [13] that was made in-house for the RFA study. MR images of a gel breast phantom were obtained and used to reconstruct an anatomical 3D CAD model for the proposed study. One advantage of using the gel material is that it is transparent at room temperature and becomes opaque when coagulated by heating. Moreover, it has a stable structure at higher temperatures and does not collapse or melt. The gel breast phantom was subsequently ablated experimentally using a commercial RFA electrode, allowing comparison between the RFA FEM simulations and actual experimental ablation.
Table 1 The recipe for tissue-mimicking phantom gel $(1 \mathrm{~L}$ with $\mathrm{pH}$ of 4.3)

\begin{tabular}{ll}
\hline Ingredients & Dosage \\
\hline Citric acid monohydrous & $24.90 \mathrm{~g}$ \\
Sodium citrate tribasic dehydrate & $23.97 \mathrm{~g}$ \\
Acrylamide & $80.00 \mathrm{~g}$ \\
$N, N^{\prime}$-methylene-bis-acrylamide & $4.00 \mathrm{~g}$ \\
BSA lyophilized powder & $20.00 \mathrm{~g}$ \\
Glycerol & $60 \mathrm{~mL}$ \\
Deionized water & Top up to $1.0 \mathrm{~L}$ \\
Initiator-activator pair & \\
$\mathrm{L}-$-ascorbic acid & $1.0 \mathrm{~g}$ \\
$1 \% \mathrm{FeSO}_{4}$ & $2.5 \mathrm{~mL}$ \\
$3 \% \mathrm{H}_{2} \mathrm{O}_{2}$ & $3.0 \mathrm{~mL}$ \\
\hline
\end{tabular}

\section{Materials and methods}

Tissue-mimicking gel breast phantom

The materials used to fabricate a tissue-mimicking gel phantom mainly included polyacrylamide mixed with bovine serum albumin (BSA), a protein used as a temperature-sensitive indicator. The mixture was subsequently polymerized using a reaction initiator-activator pair [13]. Table 1 shows the recipe for $1 \mathrm{~L}$ of phantom gel with a citrate buffer at a concentration of $0.2 \mathrm{M}$ and $\mathrm{pH}$ of 4.3. The gel properties at this $\mathrm{pH}$ are reported to mimic human soft-tissue properties with a coagulation temperature of $50{ }^{\circ} \mathrm{C}$ (gel turns opaque), although the coagulation temperature of the gel can be adjusted in the range $50-60^{\circ} \mathrm{C}$ by changing its $\mathrm{pH}$ with the addition of the citrate buffer [13]. A plastic breast mold of $400 \mathrm{~mL}$ was used to cast the gel breast phantom, and a small irregular-shaped portion of fresh baby potato (maximal dimension of $25 \mathrm{~mm}$ ) was embedded inside the phantom to simulate cancerous tissue. In order to avoid any protein denaturation resulting from the high temperature generated by the gel polymerization, the breast mold was placed in ice water during the casting process, which normally took about $2 \mathrm{~h}$.

\section{MR imaging of the breast phantom}

The gel breast phantom was then imaged in-house using a medical GE SignaHDx1.5 Magnetic resonance imaging (MRI) scanner (GE, Milwaukee, WI, USA). The phantom was positioned in the middle of an 8-channel head coil, and a total of 59 axial T2-weighted images were acquired using Fast Spin Echo MRI sequence (FSE-XL/90) with the following parameters: repetition time $(\mathrm{TR})=6,100 \mathrm{~ms}$; echo time $(\mathrm{TE})=116 \mathrm{~ms}$; field of view $(\mathrm{FOV})$ of $130 \mathrm{~mm}$; acquisitionmatrix $=256 \times 256$ and number of excitations 
$(\mathrm{NEX})=2$. The slice thickness was $2 \mathrm{~mm}$ with zero spacing and bandwidth $(\mathrm{BW})=20.8 \mathrm{kHz}$. The total acquisition time was $6 \mathrm{~min} 43 \mathrm{~s}$. Once the images were acquired, they were exported in DICOM format for further image processing and analysis.

\section{Image-based 3D CAD model}

The DICOM-compatible image slices were imported into ScanIP (v4.2, Simpleware, Ltd) for preprocessing prior to implementing the RFA FEM simulation in COMSOL Multiphysics (Comsol, UK). The imported image slices were first cleaned with Gaussian filters to remove background noise and then segmented to identify the simulated tumor and healthy tissue (i.e., the gel) by selecting image contrast boundaries. This operation enabled differentiation of tumor from the tissue by categorizing them with different masks. After threshold selection, the masks were subsequently used in a flood-fill operation during the reconstruction of the model [14]. Thereafter, the model was subjected to volumetric meshing before it was exported for use by other CAD or FEM packages. In this study, we have performed volumetric meshing and exported the mesh as both a*.mphtxt file for importing into COMSOL Multiphysics FEM package and a *.stl file for CAD design using SolidWorks (Dassault Systèmes, MA, USA).

For images of more complicated structures such as patients' breast, liver or brain, ScanIP provides various image processing tools to extract whole or particular components of the imaged anatomy. This enables the user to remove the tumor from the surrounding tissue or to identify blood vessels or other organs using a threshold operation. Once the model is converted into FEM-compatible file, it is imported to the designated software where it is integrated with a selected cancer treatment modality such as an RFA electrode for further FEM analysis.

RFA FEM simulation and experimental evaluation

A commercial radiofrequency interstitial tumor ablation (RITA) probe (StarBurst Radiofrequency Ablation, Angio-
Dynamics) was used to validate the model. The RITA probe used was a 4 tine multiprong electrode of 15 Gauge $[9,15]$. The electrode is disposable and monopolar. The electrode in its expanded state was re-drawn in COMSOL and merged with the imported 3D anatomical model, where the electrode could be rotated and moved in the model to align its position in relation to the tumor. Once the electrode was positioned in the tumor, boundary and sub-domain conditions were identified and assigned. Thereafter, the model was ready to simulate the ablation process for any duration determined by the selected treatment protocol.

A bipolar RFA needle of $3 \mathrm{~mm}$ diameter [16] was re-designed and made in-house with a reduced $25-\mathrm{mm}$ active (conducting) bipolar applicator, including a 5- $\mathrm{mm}$ insulator at the center to separate the two electrodes. This simplified bipolar needle (without internal cooling) was also used to compare results between simulated and experimental ablation.

\section{FEM modeling and RFA simulation}

COMSOL Multiphysics, which can solve the bio-heat transfer model and the electrical heating model simultaneously, was used to perform FEM analysis, thus enabling RFA simulation. In particular, Penne's equation (1) below was used to simulate heat distribution in tissues [9].

$\rho C \frac{\partial T}{\partial t}+\nabla \cdot(-k \nabla T)=\rho_{\mathrm{b}} C_{\mathrm{b}} \omega_{\mathrm{b}}\left(T_{\mathrm{b}}-T\right)+Q_{\mathrm{met}}+Q_{\mathrm{ext}}$

where $\rho$ indicates tissue density in $\left(\mathrm{kg} \mathrm{m}^{-3}\right), C$ indicates specific heat capacity of tissue at constant pressure $\left(\mathrm{J} \mathrm{kg}^{-1} \mathrm{~K}^{-1}\right)$, $k$ indicates thermal conductivity $\left(\mathrm{W} \mathrm{m}^{-1} \mathrm{~K}^{-1}\right), T$ is temperature $(\mathrm{K}), \rho_{\mathrm{b}}$ indicates density of blood $\left(\mathrm{kg} \mathrm{m}^{-3}\right), C_{\mathrm{b}}$ indicates specific heat of blood $\left(\mathrm{J} \mathrm{kg}^{-1} \mathrm{~K}^{-1}\right), \omega_{\mathrm{b}}$ indicates blood perfusion $\left(=6.4 \mathrm{e}^{-3} 1 / \mathrm{s}\right)$, and $T_{\mathrm{b}}$ indicates temperature of blood (K). Term $Q_{\text {met }}$ indicates contribution of heat due to tissue metabolic activities, and $Q_{\text {ext }}$ indicates heat source due to external RF spatial heating source $\left(\mathrm{W} \mathrm{m}^{-3}\right)[9,15]$.

At the frequencies employed in RFA (e.g., 300-500 kHz) and within the area of interest, a high electrical current density is deposited within a small radius around the active
Table 2 Material properties used in the RFA modeling $[9,13,17]$

\begin{tabular}{lclll}
\hline $\begin{array}{l}\text { Materials } \\
\text { in this } \\
\text { modeling }\end{array}$ & $\begin{array}{l}\text { Density } \\
\left(\mathrm{kg} \mathrm{m}^{-3}\right)\end{array}$ & $\begin{array}{l}\text { Specific heat } \\
\text { capacity } \\
\left(\mathrm{J} \mathrm{kg}^{-1} \mathrm{~K}^{-1}\right)\end{array}$ & $\begin{array}{l}\text { Thermal } \\
\text { conductivity } \\
\left(\mathrm{W} \mathrm{m}^{-1} \mathrm{~K}^{-1}\right)\end{array}$ & $\begin{array}{l}\text { Electrical } \\
\text { conductivity } \\
\left(\mathrm{S} \mathrm{m}^{-1}\right)\end{array}$ \\
\hline Gel & 1,069 & 3,676 & 0.59 & 0.117 \\
Breast tissue & 1,060 & 2,770 & 0.499 & 0.28 \\
Breast tumor & 1,050 & 3,770 & 0.48 & 0.71 \\
Electrode & 6,450 & 840 & 18 & $10^{8}$ \\
Trocar tip & 7,900 & 132 & 71 & $4 \times 10^{6}$ \\
Trocar base & 70 & 1,045 & 0.026 & $10^{-5}$ \\
\hline
\end{tabular}



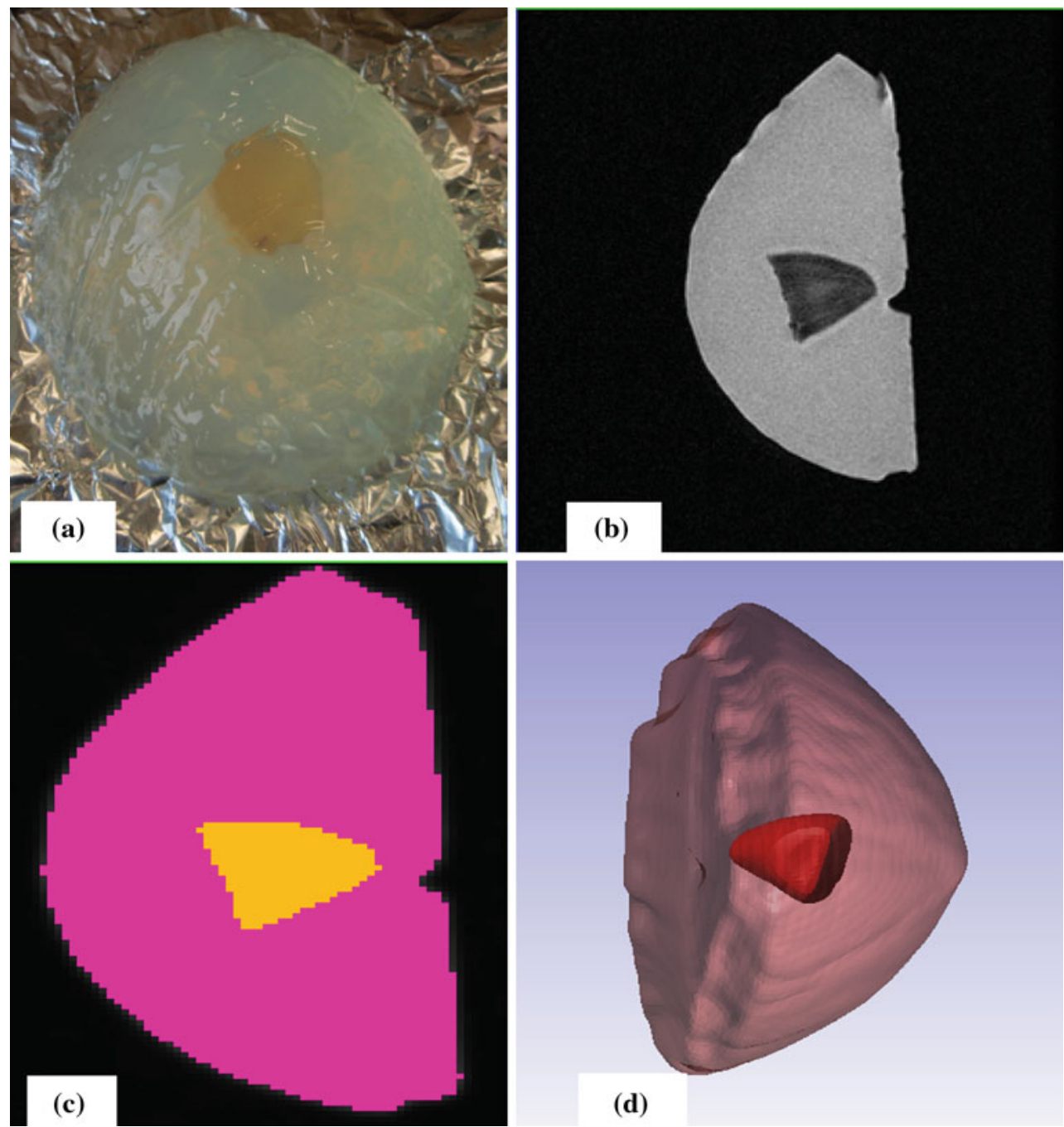

Fig. 1 Image-based 3D model: a photograph of a gl breast phantom with simulated cancerous tissue; $\mathbf{b}$ one selected MRI slice (\#32); c segmentation mask of slice \#32; d final 3D model of the gel breast phantom reconstructed from MRI slices (transparent view of the embedded simulated cancer)

electrode and the tissue can thus be simplified as purely resistive since the displacement currents are negligible. Using a quasi-static approach, the distributed heat source $Q_{\text {ext }}$ (Joule loss) is given by

$Q_{\mathrm{ext}}=J \cdot E$

where $J$ is the current density $\left(\mathrm{Am}^{-2}\right)$, and $E$ is the electric field $\left(\mathrm{Vm}^{-1}\right)$. The values of these two vectors are derived from solving Laplace's equation below

$$
\nabla \cdot(\sigma \nabla V)=0
$$

where $\sigma$ stands for electrical conductivity of tissue $\left(\mathrm{S} \mathrm{m}^{-1}\right)$, and $V$ is the applied voltage (V). Equations (1-3) were solved simultaneously by COMSOL Multiphysics with electrical and thermal material properties given in Table 2 for the tissue, tumor and electrode. Since the heat generated by tissue metabolic activities is significantly smaller than the RF heat source, we set $Q_{\text {met }}=0$. The simulation conditions include time-dependent processing with the direct (UMFPACK) linear system solver and non-symmetric matrix conditions. The simulation results display the heat distribution during the ablation process.

\section{RFA experiment}

The same phantom gel and breast phantom were used to perform experimental validation of the planned RF ablation, using the same RITA monopolar electrode and the bipolar needle, powered by a commercial electrosurgical RF generator (DRE ASG-300, DREMED, 1800 Williamson Court, Louisville, USA). The generator has the capability of delivering $120 \mathrm{~W}$ up to $1,000 \Omega$ when used in pinpoint coagulation monopolar operation and $40 \mathrm{~W}$ up to $500 \Omega$ for bipolar mode at a frequency of $490 \pm 5 \mathrm{kHz}$. 
Fig. 2 Real-time temperature plot during FEM RF ablation simulation: a 3D FEM model in Point Mode for selected points (highlighted in red); b plot of temperature increase against treatment time (s) at electrodes $\left(T_{1}\right.$ and $\left.T_{2}\right)$ and tumor edges $\left(T_{3}\right.$ and $\left.T_{4}\right)$

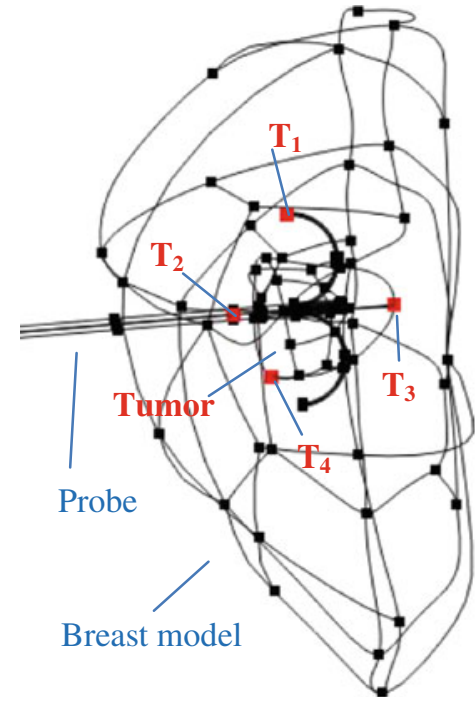

(a)

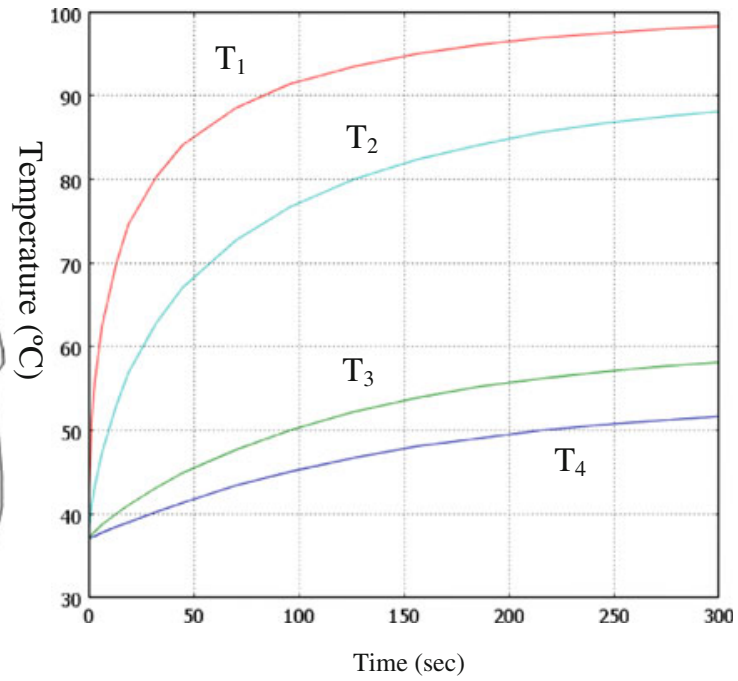

(b)

\section{Results}

Image-based 3D anatomical model

Figure 1 shows the modeling steps and results concerning fabrication of the gel breast phantom (Fig. 1a), an MR image of the phantom with one selected slice (Fig. 1b), segmentation for differentiating tissues (Fig. 1c) and reconstruction of the 3D anatomical model from the MR images (Fig. 1d). In this study, the final reconstructed 3D model (ScanIP) was exported as *.stl mesh file that was then converted into a parasolid geometry in SolidWorks. This extra step was included to reduce the number of boundary elements and use of computer memory, resulting in faster computation of the simulation.

\section{FEM RFA simulation}

The parasolid geometry of the 3D model was imported into COMSOL Multiphysics and merged with the RFA electrode geometry. Here, the electrode can be moved and rotated around to obtain the ideal position for deployment of the electrode inside the tumor (Figs. 2a, 3a). Once the insertion and deployment of the electrode within the 3D anatomy was achieved, the assembly was converted into a single composite geometry for assigning the simulation boundary conditions. The material properties shown in Table 2 were used in this modeling, and the composite geometry was meshed coarsely to reduce the computation time. The final mesh consists of 26,459 elements. Simulated RF ablation was then performed with these tissue properties and the selected power and treatment time.

The ablation simulation stopped automatically when one of the following two pre-set conditions was achieved: (i) the maximal temperature reached a selected threshold (e.g., $100^{\circ} \mathrm{C}$ ), or (ii) the RF energy dose was completed. A real-time temperature plot at selected points on the model (Fig. 2a) could be displayed (Fig. 2b) to assist monitoring of the ablation process, for example, to monitor when the temperature at the tumor edges had increased above $50{ }^{\circ} \mathrm{C}$ $\left(T_{3}\right.$ and $\left.T_{4}\right)$. The simulation showed that at this applied RF energy of $7.5 \mathrm{~kJ}$, the maximal temperature at electrode $T_{1}$ was below $100^{\circ} \mathrm{C}$. This is important because of the need to avoid tissue carbonization that impedes or even blocks the RF current and heat transfer into the tissue [10].

In addition to choosing probe trajectories for deployment and keeping maximal ablation temperature below $100^{\circ} \mathrm{C}$, optimization of the applied energy (i.e., the power and treatment time) in the simulation was dictated by the following criteria: (i) total ablation of tumor and (ii) minimal collateral damage to the surrounding healthy tissue [18]. One optimal simulation case is outlined below. The RITA probe was deployed at a preferred location in the tumor (Fig. 3a), at an applied energy of $7.5 \mathrm{~kJ}$ (i.e., $25 \mathrm{~W}$ and $300 \mathrm{~s}$ ). We estimated the ablated tissue boundary using an iso-thermal surface plot for a tissue temperature of $50^{\circ} \mathrm{C}$ and above (Fig. 3b). From visual inspection, it is evident that the ablation volume encompasses the entire tumor (criterion 1). As for criterion 2, given the irregular tumor shape and the limitation of the fixeddistance probe electrode deployment, healthy tissue might be damaged to ensure a safe margin.

The bipolar needle probe was deployed in the tumor (Fig. 3c), and one applied energy dose of $2.25 \mathrm{~kJ}$ (i.e., $7.5 \mathrm{~W}$ and $300 \mathrm{~s}$ ) was chosen after the optimization process as described earlier. Again the ablated tissue volume was estimated using an iso-thermal surface plot for a tissue temperature of $50^{\circ} \mathrm{C}$ and above (Fig. 3d). In this instance, visual 
Fig. 3 Anatomical 3D

modeling and simulation of RFA using COMSOL

Multiphysics with breast tissue and tumor material properties (Table 2): a composite model geometry with RITA monopolar probe inserted and deployed into the simulated tumor tissue; $\mathbf{b}$ with an applied power of $25 \mathrm{~W}$, the iso-thermal surface plot of $50{ }^{\circ} \mathrm{C}$ indicating the ablation volume at $5 \mathrm{~min}$, which encompassed whole tumor with a safe margin; $\mathbf{c}$ bipolar RFA needle placed within the model; and $\mathbf{d}$ bipolar ablation zone with iso-thermal surface plot at $50{ }^{\circ} \mathrm{C}$ : after treatment for $300 \mathrm{~s}$ at $7.5 \mathrm{~W}$, the iso-thermal surface did not encompass whole tumor

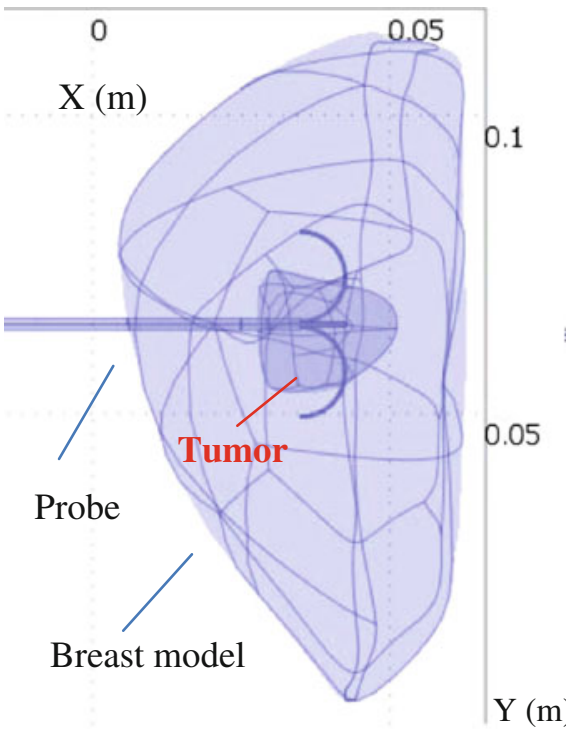

(a)
Color scale $\left({ }^{\circ} \mathrm{C}\right)$

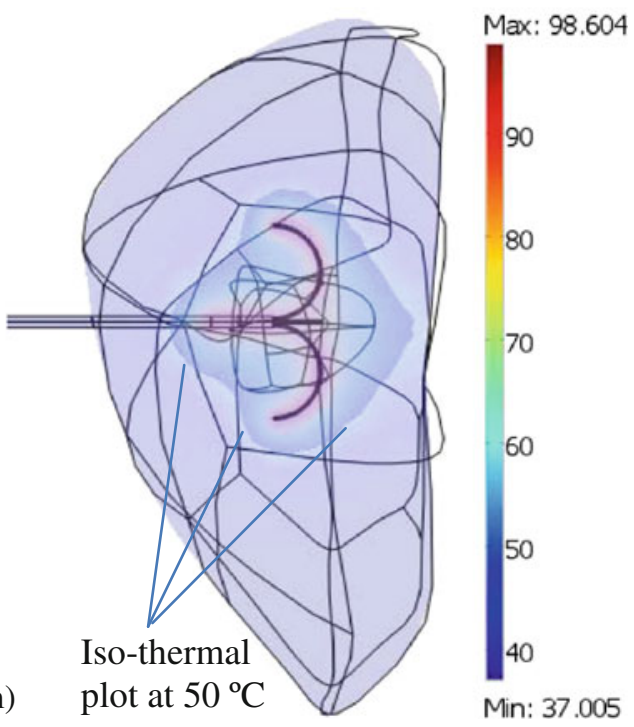

(b)

Color scale $\left({ }^{\circ} \mathrm{C}\right)$

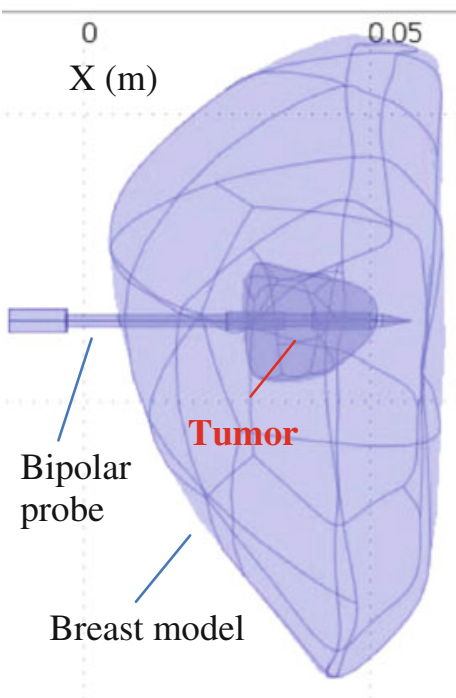

(c)

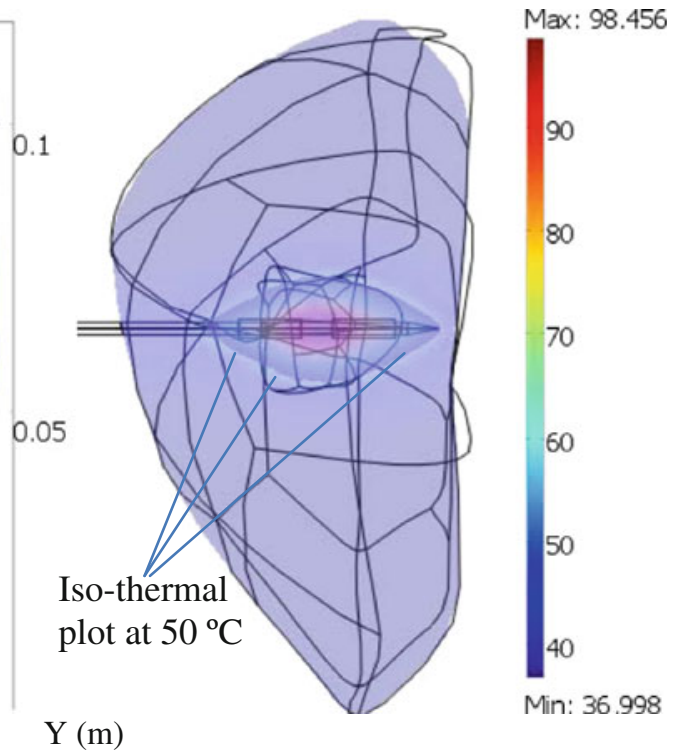

(d) inspection shows that the ablation volume did not cover the entire tumor (criterion 1). Two bipolar needles were needed to encompass the full extent of the tumor [4].

The simulation on the model was performed using both breast tissue and tumor tissue properties and included the effect of perfusion in both the domains. Other biophysical properties could be simulated in the future for the construction of real patient models, which could include blood vessels and the heat-sink effect due to blood flow at or near the tumor site. In the present study, FEM modeling was followed by repeating the simulation procedure on non-cancerous breast tissue. This was also used to evaluate the performance of the electrode (see results in Table 3).

Experimental validation

For this, the same gel phantom and the RITA electrode were used (Fig. 4a), and the environmental conditions were kept the same. The power delivered to the electrode for sufficient ablation was found to be $25 \mathrm{~W}$ that is the same as that in 
Table 3 Volume and size measurements of RFA ablation versus FEM analysis in two RFA needles

\begin{tabular}{llllr}
\hline & Applied energy $Q(\mathrm{~kJ})$ & Longitudinal ablation size $L(\mathrm{~cm})$ & Transversal ablation size $W(\mathrm{~cm})$ & $\begin{array}{l}\text { Volume* } \\
V\left(\mathrm{~cm}^{3}\right)\end{array}$ \\
\hline Ablation-I & 7.5 & 3.40 & 4.40 & 34.45 \\
FEM-I & 7.5 & 3.45 & 4.35 & 34.16 \\
Ablation-II & 2.25 & 3.4 & 1.49 & 3.94 \\
FEM-II & 2.25 & 3.44 & 1.48 & 3.95 \\
\hline
\end{tabular}

Two RFA needles: I refers to RITA monopolar needle, and II refers to the bipolar needle

* Volume is calculated using the formula $\left(V=(\pi / 6) L W^{2}\right)$ from [19] for ellipsoid volume

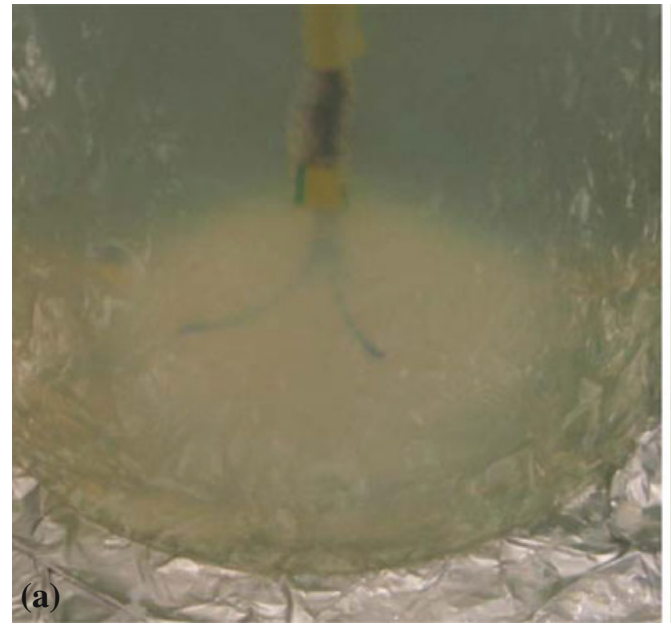

Fig. 4 RFA experimental validation against FEM simulation, using the same RFA electrode and gel phantom and similar RF power and treatment time: a the RITA electrode inserted and deployed into the gel

FEM simulation in the model. Miniature thermocouple sensors were used to monitor real-time temperature changes during ablation, and these too were similar (data not shown) to the simulation temperatures shown in Fig. 2b. The ablation was carried out over $300 \mathrm{~s}$, and the ablated gel region (which opacified at temperatures over $50^{\circ} \mathrm{C}$ ) was dissected to measure its dimension (Fig. 4b). In brief, following ablation and withdrawal of the probe, the gel phantom was cut along the longitudinal plane, passing through the probe axis. (Additional slices can be cut along the transverse plane when the ablation geometry is not symmetrical due to local variations caused by proximity to a large blood vessel. However, this was not the case for this gel phantom study.) The ablation zone was determined from the maximal transverse ( $W$ in $\mathrm{cm}$ ) and longitudinal ( $L$ in $\mathrm{cm})$ dimensions: the volume $\left(V\right.$ in $\left.\mathrm{cm}^{3}\right)$ was calculated using the formula for ellipsoid volume, that is, $V=(\pi / 6) L W^{2}[16]$.

Table 3 lists the volume and size measurements of RFA ablation, together with FEM simulation results. Group I (Ablation-I and FEM-I) refers to results using RITA monopolar probe; while group II, the data for the bipolar needle. The volume of ablation achieved is very similar to that obtained by FEM analysis. Also the data confirm that the

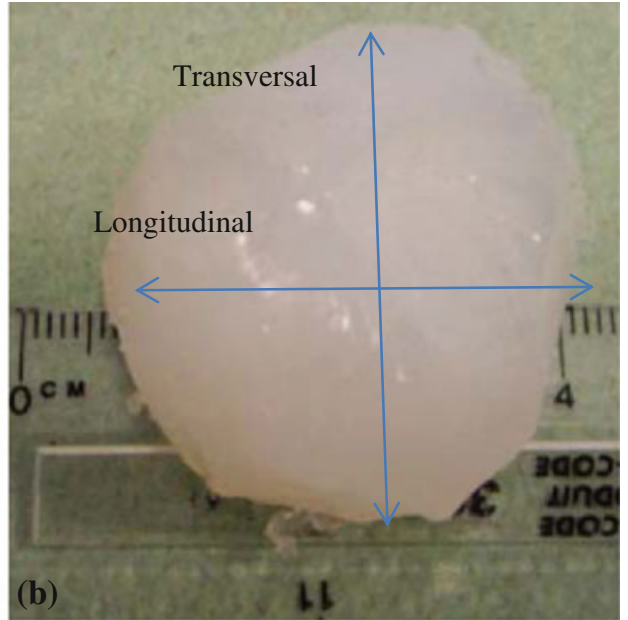

phantom and RFA in progress with ablated gel becoming opaque; $\mathbf{b}$ a completed ablation at $5 \mathrm{~min}$ using $25 \mathrm{~W}$ : the ablated region was excised for dimension measurement

RITA RF electrode can completely ablate the tumor, as distinct from the bipolar needle, which in the study could not achieve completed ablation that would require multiple bipolar needles [20].

We recognize that the model used in the present study is simple and requires further development to a more realistic model based on real clinical MR or CT images in order to be useful clinically as it would need to simulate more complicated geometries in surrounding tissues and organs [21]. There are also limitations inherent to ex-vitro experiments especially as the gel phantom did not contain any vessels to simulate blood flow and perfusion. However, there have been reported studies that compared ex-vivo and in-vivo ablation. Zlotta et al. [22] reported that in the in-vivo procedure, the size of the lesions in human renal cancer was comparable to the forecast size derived from an ex-vivo model consisting of a harvested kidney with perfusion. Cha et al. [23] reported that their laboratory studies using internally cooled electrodes in animal models and found reduced (up to 1/3) ablation sizes in in-vivo studies $(n=16)$ when compared to the ex-vivo experiments $(n=30), 2.62 \times 2.0 \mathrm{~cm}$ for invivo and $3.01 \times 2.62 \mathrm{~cm}$ for ex-vivo, respectively. Hence, an advanced patient-specific 3D CAD/FEM model, with 
real-time feedback of in-situ treatment temperature and with continuous updating of temperature-dependent tissue physical properties, is needed for more accurate simulation of RFA pre-treatment planning designed to improve the efficacy of clinical RFA ablation of tumors.

\section{Conclusions}

We have successfully developed and validated an imagebased 3D modeling of RFA. This pilot study indicates that this approach to overcome the current problems encountered in clinical RFA is promising but requires further development with the use of clinical images obtained by CT or MRI to construct patient-specific models for RFA planning and simulation with a view to optimizing treatment efficacy.

Acknowledgments This article presents independent research partially commissioned by the National Institute for Health Research (NIHR) under Invention for Innovation (i4i) Programme. The views expressed in this article are those of the authors and not necessarily those of the NHS, the NIHR or the Department of Health.

\section{Conflict of interest None.}

\section{References}

1. Wood BJ, Ramkaransingh JR, Fojo T, Walther MM, Libutti SK (2002) Percutaneous tumor ablation with radiofrequency. Cancer 94:43-451

2. Lau WY, Leung TW, Yu SC, Ho SK (2003) Percutaneous local ablative therapy for hepatocellular carcinoma: a review and look into the future. Ann Surg 237:171-179

3. Morrow M (2009) Minimally invasive surgery for breast cancer. Br Med J 338

4. Fornage BD, Sneige N, Ross MI, Mirza AN, Kuerer HM, Edeiken BS, Ames FC, Newman LA, Babiera GV, Singletary SE (2004) Small $(<$ or $=2-\mathrm{cm})$ breast cancer treated with US-guided radiofrequency ablation: feasibility study. Radiology 231:215-224

5. Okhunov Z, Roy O, Duty B, Waingankar N, Herati A, Morgenstern N, Sheikh-Fayyaz S, Kavoussi LR (2012) Clinical evaluation of a novel bipolar radiofrequency ablation system for renal masses. BJU Int

6. Payne S, Flanagan R, Pollari M, Alhonnoro T, Bost C, O’Neill D, Peng T, Stiegler P (2011) Image-based multi-scale modelling and validation of radio-frequency ablation in liver tumours. Philos Trans R Soc A Math Phys Eng Sci 369:4233-4254

7. dos Santos I, Haemmerich D, Pinheiro C, da Rocha A (2008) Effect of variable heat transfer coefficient on tissue temperature next to a large vessel during radiofrequency tumor ablation. BioMed Eng OnLine 7:21

8. Rieder C, Kroeger T, Schumann C, Hahn HK (2011) GPU-based real-time approximation of the ablation zone for radiofrequency ablation. IEEE Trans Vis Comput Graph 17:1812-1821
9. Tungjitkusolmun S, Staelin ST, Haemmerich D, Jang-Zern T, Hong C, Webster JG, Lee FT Jr, Mahvi DM, Vorperian VR (2002) Three-dimensional finite-element analyses for radiofrequency hepatic tumor ablation. IEEE Trans Biomed Eng 49:3-9

10. Barauskas R, Gulbinas A, Vanagas T, Barauskas G (2008) Finite element modeling of cooled-tip probe radiofrequency ablation processes in liver tissue. Comput Biol Med 38:694-708

11. Datta A, Bansal V, Diaz J, Patel J, Reato D, Bikson M (2009) Gyri-precise head model of transcranial direct current stimulation: improved spatial focality using a ring electrode versus conventional rectangular pad. Brain Stimul 2:201-207, e201

12. Fuentes D, Cardan R, Stafford RJ, Yung J, Dodd GD 3rd, Feng Y (2010) High-fidelity computer models for prospective treatment planning of radiofrequency ablation with in vitro experimental correlation. J Vasc Interv Radiol 21:1725-1732

13. Bu-Lin Z, Bing H, Sheng-Li K, Huang Y, Rong W, Jia L (2008) A polyacrylamide gel phantom for radiofrequency ablation. Int $\mathrm{J}$ Hyperthermia 24:568-576

14. Rossi MA, Stebbins G, Murphy C, Greene D, Brinker S, Sarcu D, Tenharmsel A, Stoub T, Stein MA, Hoeppner TJ, Byrne RW, Moseley ME, Bammer RA, Bild S, Dennis J, Arnett N, Balabanov A, Bergen D, Kanner AM, Smith MC (2010) Predicting white matter targets for direct neurostimulation therapy. Epilepsy Res 91:176-186

15. Haemmerich D, Webster JG (2005) Automatic control of finite element models for temperature-controlled radiofrequency ablation. Biomed Eng Online 4:42

16. Ritz J-P, Lehmann KS, Isbert C, Reissfelder C, Albrecht T, Stein $\mathrm{T}$, Buhr HJ (2006) In-vivo evaluation of a novel bipolar radiofrequency device for interstitial thermotherapy of liver tumors during normal and interrupted hepatic perfusion. J Surg Res 133:176-184

17. Duck FA (1990) Physical properties of tissue: a comprehensive reference book. Academic Press, London

18. Butz T, Warfield S, Tuncali K, Silverman S, van Sonnenberg E, Jolesz F, Kikinis R (2000) Pre- and intra-operative planning and simulation of percutaneous tumor ablation. In: Delp S, DiGoia A, Jaramaz B (eds) Medical image computing and computer-assisted intervention-MICCAI 2000. Springer, Berlin, pp 395-416

19. Häcker A, Vallo S, Weiss C, Grobholz R, Stein T, Knoll T, Michel MS (2006) Bipolar and multipolar radio frequency ablation with resistance controlled power output: standardized ex vivo kidney tissue evaluation. J Urol 175:1122-1126

20. Stoffner R, Kremser C, Schullian P, Haidu M, Widmann G, Bale RJ (2012) Multipolar radiofrequency ablation using 4-6 applicators simultaneously: a study in the ex vivo bovine liver. Eur J Radiol

21. Patterson EJ, Scudamore CH, Owen DA, Nagy AG, Buczkowski AK (1998) Radiofrequency ablation of porcine liver in vivo: effects of blood flow and treatment time on lesion size. Ann Surg 227:559-565

22. Zlotta AR, Wildschutz T, Raviv G, Peny MO, van Gansbeke D, Noel JC, Schulman CC (1997) Radiofrequency interstitial tumor ablation (RITA) is a possible new modality for treatment of renal cancer: ex vivo and in vivo experience. J Endourol 11:251-258

23. Cha J, Choi D, Lee MW, Rhim H, Kim YS, Lim HK, Yoon JH, Park CK (2009) Radiofrequency ablation zones in ex vivo bovine and in vivo porcine livers: comparison of the use of internally cooled electrodes and internally cooled wet electrodes. Cardiovasc Intervent Radiol 32:1235-1240 